
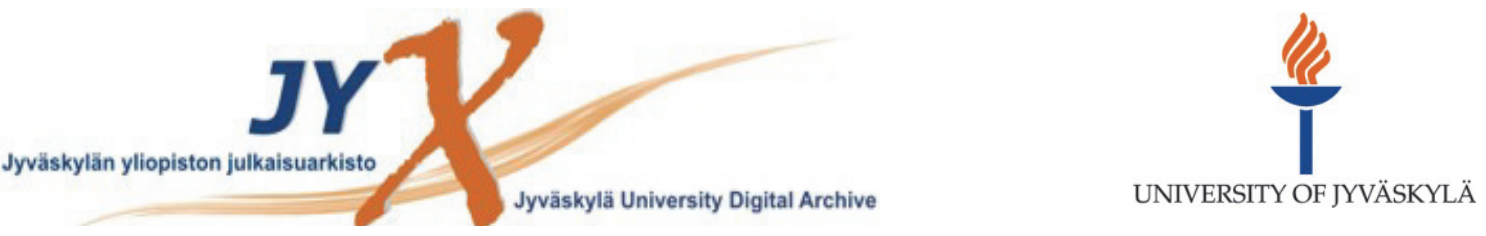

This is an electronic reprint of the original article. This reprint may differ from the original in pagination and typographic detail.

\author{
Author(s): Guadilla, V.; Algora, A.; Tain, J.L.; Agramunt, J.; Äystö, Juha; Briz, A.; Cano-Ott, D.; \\ Cucoanes, A.; Eronen, Tommi; Estienne, M.; Faillot, M.; Fraile, L.M.; Ganioglu, E.; \\ Gelletly, W.; Gorelov, Dmitry; Hakala, Jani; Jokinen, Ari; Jordan, D.; Kankainen, Anu; \\ Kolhinen, Veli; Koponen, Jukka; Lebois, M.; Martinez, T.; Monserrate, M.; Montaner- \\ Pizá, A.; Moore, lain; Nácher, E.; Orrigo, S.; Penttilä, Heikki; Podolyak, Zs.; Pohjalainen, \\ Title: $\quad$ First experiment with the NUSTAR/FAIR Decay Total Absorption $\psi$-Ray Spectrometer \\ (DTAS) at the IGISOL IV facility
}

Year: $\quad 2016$

Version:

Please cite the original version:

Guadilla, V., Algora, A., Tain, J.L., Agramunt, J., Äystö, J., Briz, A., Cano-Ott, D., Cucoanes, A., Eronen, T., Estienne, M., Faillot, M., Fraile, L.M., Ganioglu, E., Gelletly, W., Gorelov, D., Hakala, J., Jokinen, A., Jordan, D., Kankainen, A., . . Zakari-Issoufou, A.-A. (2016). First experiment with the NUSTAR/FAIR Decay Total Absorption $\gamma$-Ray Spectrometer (DTAS) at the IGISOL IV facility. Nuclear Instruments and Methods in Physics Research Section B: Beam Interactions with Materials and Atoms, 376, 334337. https://doi.org/10.1016/j.nimb.2015.12.018

All material supplied via JYX is protected by copyright and other intellectual property rights, and duplication or sale of all or part of any of the repository collections is not permitted, except that material may be duplicated by you for your research use or educational purposes in electronic or print form. You must obtain permission for any other use. Electronic or print copies may not be offered, whether for sale or otherwise to anyone who is not an authorised user. 


\title{
First experiment with the NUSTAR/FAIR Decay Total Absorption $\gamma$-Ray Spectrometer (DTAS) at the IGISOL IV facility
}

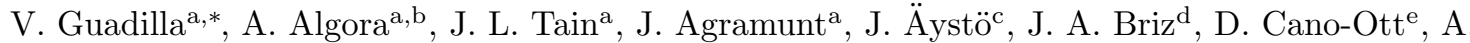 \\ Cucoanes $^{\mathrm{d}}$, T. Eronen ${ }^{\mathrm{c}}$, M. Estienne ${ }^{\mathrm{d}}$, M. Fallot ${ }^{\mathrm{d}}$, L. M. Fraile ${ }^{\mathrm{f}}$, E. Ganioglu ${ }^{\mathrm{g}}$, W. Gelletly ${ }^{\mathrm{h}}$, D. Gorelov ${ }^{\mathrm{c}}$,

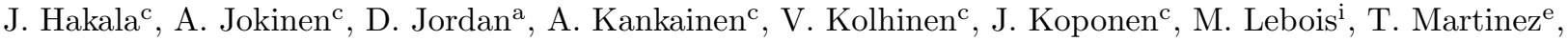 \\ M. Monserrate ${ }^{\mathrm{a}}$, A. Montaner-Pizáa ${ }^{\mathrm{a}}$, I. Moore ${ }^{\mathrm{c}}$, E. Nácher ${ }^{\mathrm{j}}, \mathrm{S}_{\text {. Orrigo }}^{\mathrm{a}}$, H. Penttiläc ${ }^{\mathrm{C}}$ Zs. Podolyak ${ }^{\mathrm{h}}$, I. \\ Pohjalainen $^{c}$, A. Porta ${ }^{\mathrm{d}}$, P. Regan ${ }^{\mathrm{h}}$, J. Reinikainen ${ }^{\mathrm{c}}$, M. Reponen ${ }^{\mathrm{c}}$, S. Rinta-Antila ${ }^{\mathrm{c}}$, B. Rubio ${ }^{\mathrm{a}}$, K. \\ Rytkönen $^{c}$, T. Shiba ${ }^{\mathrm{d}}$, V. Sonnenschein ${ }^{\mathrm{c}}$, A. A. Sonzogni ${ }^{\mathrm{k}}$, E. Valencia ${ }^{\mathrm{a}}$, V. Vedia ${ }^{\mathrm{f}}$, A. Voss $^{\mathrm{c}}$, J. N. \\ Wilson $^{\mathrm{i}}$, A. -A. Zakari-Issoufou ${ }^{\mathrm{d}}$

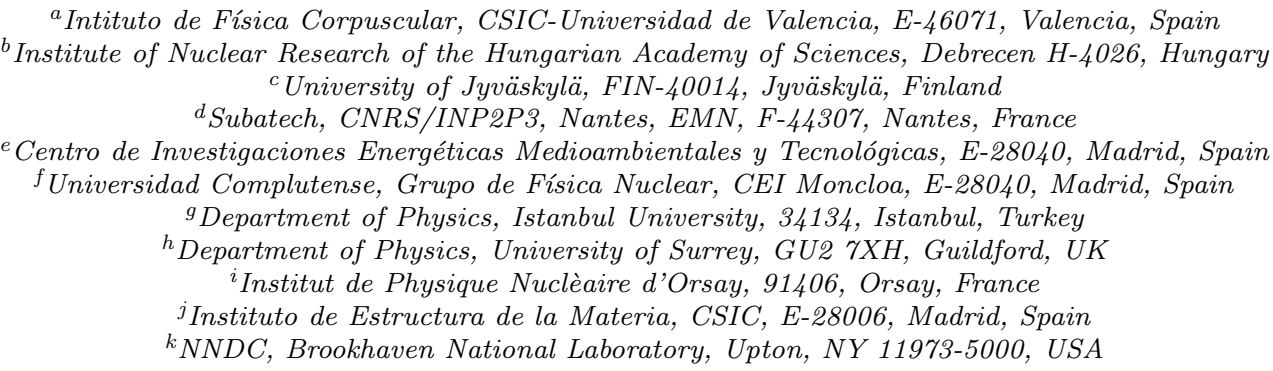

\begin{abstract}
The new Decay Total Absorption Spectrometer (DTAS) has been commissioned with low energy radioactive beams at the upgraded IGISOL IV facility. The DTAS is a segmented detector composed of up to 18 $\mathrm{NaI}(\mathrm{Tl})$ crystals and it will be a key instrument in the DESPEC experiment at FAIR. In this document we report on the experimental setup and the first measurements performed with DTAS at IGISOL. The detector was characterized by means of MC simulations, and this allowed us to calculate the response function of the spectrometer and analyse the first cases of interest.
\end{abstract}

Keywords: $\beta$ decay, total absorption $\gamma$-ray spectrometer, exotic nuclei, ISOL facilities, $\beta$-delayed neutron emitters

\section{Introduction}

In Nuclear Physics, the $\beta$-strength distribution can be used to study nuclear structure since it is sensitive to the overlap of the initial and final wave functions of the parent and daughter states. The relation of the $\beta$-intensity $I_{\beta}$ to a state of energy $E_{x}$ with the $\beta$-strength $S_{\beta}$ and the squared theoretical matrix elements $B$ is given by:

$$
S_{\beta}\left(E_{x}\right)=\frac{I_{\beta}\left(E_{x}\right)}{f\left(Q_{\beta}-E_{x}\right) T_{1 / 2}}=\frac{1}{C} B\left(E_{x}\right)
$$

\footnotetext{
* Corresponding author

Email address: victor.guadilla@ific.uv.es (V. Guadilla)
}

Preprint submitted to Nuclear Instruments and Methods B where $T_{1 / 2}$ is the half life of the parent nucleus, $f\left(Q_{\beta}-E_{x}\right)$ is the statistical Fermi function and $C$ is a constant.

Total absorption $\gamma$-ray spectroscopy (TAGS) has been shown to be an effective tool to determine $\beta$ decay intensity distributions for nuclei far from the valley of $\beta$ stability [1, 2, 3]. In contrast with high resolution spectroscopy, where HPGe detectors are used to detect individual $\gamma$ rays, TAGS aims to detect the full $\beta$-delayed electromagnetic cascade, avoiding the systematic error known as the Pandemonium effect 4 related to the modest efficiency of the HPGe detectors, that shifts the apparent $I_{\beta}$ distribution to lower excitation energies. The main

June 16, 2016 
requirement for such a detector is to maximize the efficiency by using large scintillator crystals to cover a solid angle of $\sim 4 \pi$. For an ideal detector, with $100 \% \gamma$-ray efficiency, the measured energy spectrum would provide directly the $\beta$-intensity distribution convoluted with the instrumental resolution. Bearing this in mind a new spectrometer has been designed and constructed [5] for the study of exotic nuclear species at the focal plane of the FAIRNUSTAR Super Fragment Separator in the DESPEC experiment [6. Such a system will provide information relevant to studies of nuclear structure and nuclear astrophysics.

The Decay Total Absorption $\gamma$-Ray Spectrometer (DTAS) consists of a maximum of 18 rectangular $\mathrm{NaI}(\mathrm{Tl})$ crystals with dimensions $150 \mathrm{~mm} \times$ $150 \mathrm{~mm} \times 250 \mathrm{~mm}$. In the configuration foreseen for FAIR [5], a sixteen-module assembly will be coupled to the Advanced Implantation Detector Array (AIDA) [7]. In the present work, however, the full eighteen-module assembly, depicted in Figure 1 , has been used allowing us to place the beam pipe and a HPGe ancillary detector inside. This configuration exhibits a $65 \%$ photopeak efficiency at $1 \mathrm{MeV}$.
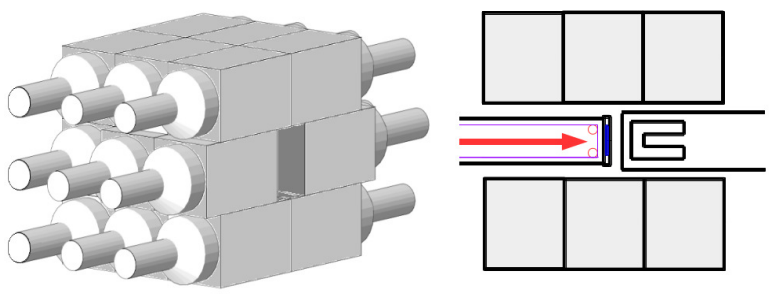

Figure 1: Schematic drawing of the DTAS detector in the eighteen-module configuration (left) and a lateral cut through the setup used in the measurements (right) where the beampipe, the tape system, the plastic detector and the Ge detector can be seen.

\section{Experiment}

\subsection{Motivation}

The experiment focused on the study of uranium fission fragments with the TAGS technique. Most of these nuclei have large $Q_{\beta}$ values, and are suspected to suffer from the Pandemonium effect. Many of them are involved in reactor decay heat summation calculations [1, 2] and are important contributors to the antineutrino spectra from reactors 8 . There are also a few cases of interest in relation to $\beta$-delayed neutron emission, in which the neutron separation energy $S_{n}$ in the daughter nucleus is lower than the decay energy window $Q_{\beta}$, and competition between $\gamma$ and neutron emission from neutron unbound states can be studied [9, 10].

\subsection{Experimental setup}

The first experiment with DTAS was performed at the upgraded IGISOL IV facility of the University of Jyväskylä, Finland [11] in February 2014. Proton beams from the MCC30 cyclotron were used to induce fission in a natural Uranium target. At IGISOL the fission products recoil out of the target and stop in a gas (usually helium). Then, a jet gas flow transports them through a differential pumping system directly into the first stage of the mass separator.

One advantage of the IGISOL facility is the possibility to use the JYFLTRAP double Penning trap to separate out isobaric contaminants [12. In our experiment, after the purification in JYFLTRAP the activity was implanted on a tape placed in vacuum at the centre of DTAS. The tape transport system runs in cycles which were optimised for each nucleus. The DTAS was surrounded by shielding composed of stainless steel sheets, lead bricks and aluminium, which served to reduce the background counting rate by one order-of-magnitude. The whole assembly, detector plus shielding, laid on an aluminum table with two rails that enable to separate the DTAS in two independent structures, each of them containing half of the crystals. The full experimental arrangement is shown in Figure 2 .

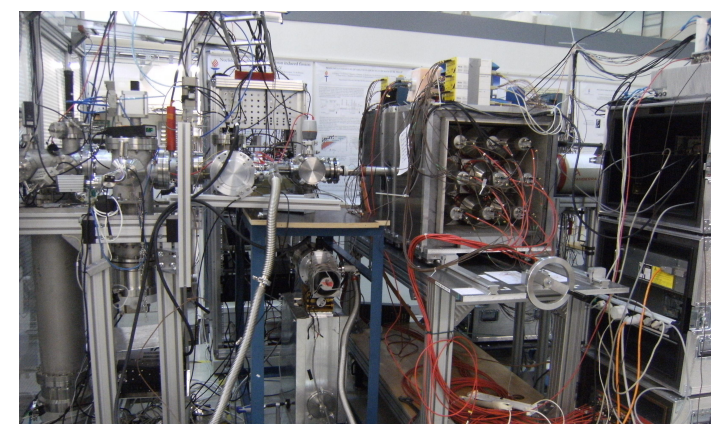

Figure 2: Setup at IGISOL with the DTAS surrounded by the shielding. The beam pipe enters from the left side and the HPGe detector from the right side (see Figure 1 right).

Both anode and last dynode signals from each phototube were shaped with Mesytec MSI-8p 
preamplifiers. Anode signals were added to provide a timing signal using a constant fraction discriminator. Dynode signals were further processed with Mesytec MSCF-16 shapers before being sent to the ADC to reconstruct the total absorption spectrum. Data were collected simultaneously in a conventional triggered acquisition system and in a new trigger-less system. Each individual spectrum covered a range of $15 \mathrm{MeV}$ with a threshold of $80 \mathrm{keV}$.

A method to correct changes in the photomultiplier gain has been developed based on an external reference detector. This was a 3" $\times 3$ " welltype $\mathrm{NaI}(\mathrm{Tl})$ crystal with a weak ${ }^{137} \mathrm{Cs}$ source inside. This detector and all DTAS modules were illuminated with a $6010 \mathrm{BNC} 490 \mathrm{~nm}$ light pulser source through a bundle of borosilicate glass fibers. The reference detector was employed to monitor the light source thus allowing a gain correction in all phototubes. This was essential to avoid distortions in the spectra and we achieved an energy resolution of $8.8 \%$ at $662 \mathrm{keV}$.

In order to eliminate background events in the spectrometer, coincidences with $\beta$ particles were required. A $3 \mathrm{~mm}$ plastic scintillator detector with $30 \%$ detection efficiency was placed in front of the tape, where the activity was implanted. We chose a segmented $2 \times 2$ Multianode Hamamatsu PMT R7600U-M4 for the plastic detector. By requiring coincidences between the segments we could reduce the noise level and set an energy threshold of $70 \mathrm{keV}$.

The setup was completed with a HPGe detector placed behind the $\beta$ plastic detector. It was used to identify possible contamination coming from the activity of the decay chain.

\section{Detector performance}

In order to determine a $\beta$-intensity distribution from the measured spectrum, a de-convolution has to be done with the spectrometer response to the decay [13]. For this purpose we have to solve the inverse problem represented by:

$$
d_{i}=\sum_{j} R_{i j} f_{j}
$$

where $d_{i}$ is the number of counts in channel i, $f_{j}$ is the number of events that fed level $\mathrm{j}$ in the daughter and $R_{i j}$ response function of the detector that represents the probability that feeding to the level $\mathrm{j}$ gives a count in channel $\mathrm{i}$ of the spectrum.

A method to de-convolute the feeding distribution has been developed [14] and successfully applied to previous measurements [1, 2, 3, 15. Since the response function is unique to each detector and each decay scheme, it has to be calculated via MC codes, with the geometry and the physics involved in the detection process. Hence, the key step in the characterization consists of simulating calibration sources to obtain the best match with the corresponding experimental measurements. For this purpose the package Geant4 [16] has been used and the detailed geometry of the DTAS, the ancillary detectors and the beam pipe has been included. In order to compare the experimental measurements and the simulations, any source of contamination has to be subtracted. In the case of the calibration sources apart from subtracting the environmental background, we have calculated the effect of random summing of signals from different detector modules and of pileup in a single detector module 17. A Monte Carlo procedure to calculate both contributions based on the random superposition of two stored events within the ADC gate length was developed for previous works [10] and has been successfully applied here. As can be observed in Figure 3, we obtain an excellent reproduction of the measured spectrum with MC simulations.

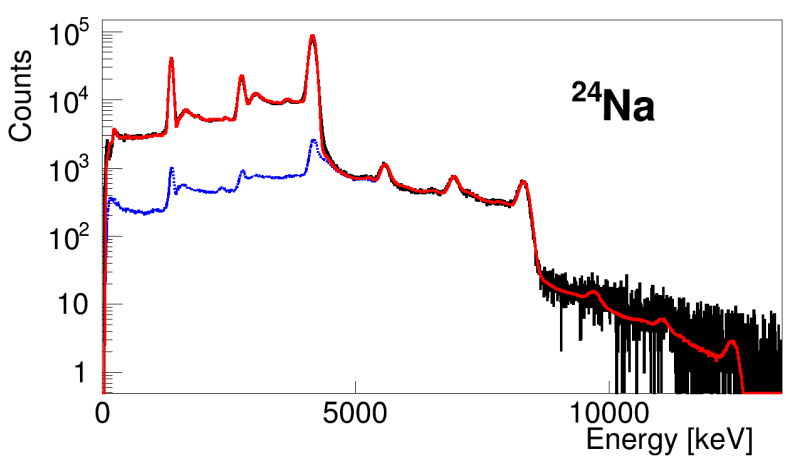

Figure 3: ${ }^{24} \mathrm{Na}$ source produced at IGISOL. The background subtracted experimental spectrum (black) is compared with the $\mathrm{MC}$ (red) after adding the summing-pileup (blue).

\section{Selected case: ${ }^{137} I$}

As an example, we present here a preliminary analysis of the decay of ${ }^{137} \mathrm{I}$, an important $\beta$-delayed neutron emitter with $P_{n}=7.14 \%$ 
and $T_{1 / 2}=24.5 \mathrm{~s}$. In this case $Q_{\beta}=6.027 \mathrm{MeV}$ and $S_{n}=4.025 \mathrm{MeV}$, and it is known from highresolution experiments [18, 19] that $\gamma$ emission competes with neutron emission above $S_{n}$. The rate was 350 nuclei/s and the decay of the daughter, ${ }^{137} \mathrm{Xe}$, with $T_{1 / 2}=3.83 \mathrm{~min}$, was also measured in order that it can be subtracted as a contaminant. Apart from the summing-pileup, an important source of contamination in this case are the neutrons released in the decay process that interact with DTAS. This contribution has been evaluated with Geant4 in the line of previous works [5, 10, 20, using the ENDF-VII0 library and a modified neutron capture cascade generator. In particular, we observe in Figure 4 a peak at $6.83 \mathrm{MeV}$ which comes from neutron capture in ${ }^{127} \mathrm{I}$, while below $2 \mathrm{MeV}$ inelastic interactions dominate.

The detector response to the decay was calculated using the known decay scheme at low excitation energies and the nuclear statistical model at high excitation energies. Figure 4 shows the quality of the reproduction of the measured spectrum with this response. From this preliminary analysis we obtain $8.7(10) \% \beta$ intensity followed by $\gamma$ emission above $S_{n}$, in contrast with the $2.75 \%$ reported in the ENSDF database 21. The situation is similar to that found in the decay of ${ }^{87} \mathrm{Br}[10$. The allowed decay of ${ }^{137} \mathrm{I}$ populates positive parity states with $\mathrm{J}=5 / 2,7 / 2,9 / 2$ and the decay of such states to the $0^{+}$g.s. of ${ }^{136} \mathrm{Xe}$ requires the emission of neutrons with large orbital angular momentum and is, therefore, hindered. In addition, a sizeable Pandemonium effect in the average $\gamma$ and $\beta$ decay energies is also found, as reported in Table 1 .

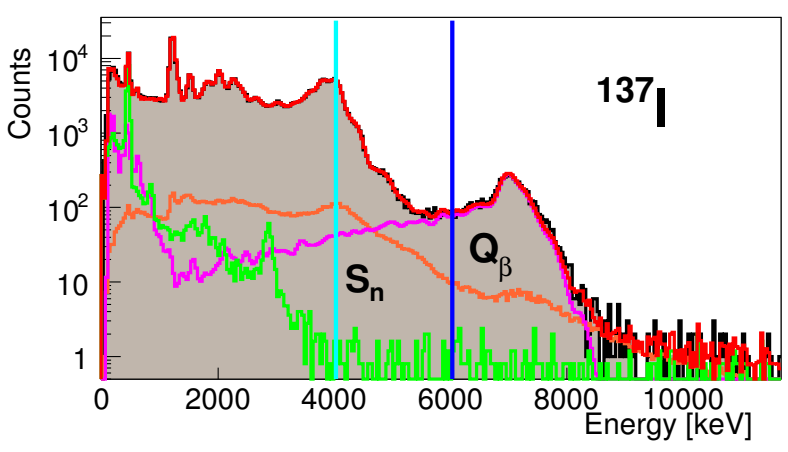

Figure 4: ${ }^{137}$ I analysis with relevant histograms: experiment (black), daughter decay (green), summing-pileup (orange), $\beta$-delayed neutron decay (pink) and reconstructed spectrum (red).

\begin{tabular}{|c|c|}
\hline ENSDF [keV] & DTAS [keV] \\
\hline $\bar{E}_{\gamma}=1075$ & $\bar{E}_{\gamma}=1335(40)$ \\
\hline $\bar{E}_{\beta}=1964$ & $\bar{E}_{\beta}=1842(20)$ \\
\hline
\end{tabular}

Table 1: Mean $\beta$ and $\gamma$ energies obtained from the preliminary TAGS analysis compared with values from ENSDF.

\section{Conclusions}

The DTAS detector designed for DESPEC (FAIR) has been commissioned in the eighteenmodule assembly with low energy radioactive beams at IGISOL. These measurements allowed us to perform a careful characterization of the DTAS detector response, as well as of the $\beta$ plastic detector. A good agreement between calibration sources and MC simulations has been achieved, so that we are able to calculate the response function of the decays of interest, and carry out the analysis of the measured fission products. The overall good performance of the detector and the gain stabilization system have been demonstrated, and we have achieved a good understanding of the detector response. This is an important step towards the application of DTAS at FAIR with high energy beams.

\section{Acknowledgements}

This work has been supported by the Spanish Ministerio de Economía y Competitividad under the FPA2011-24553, the AIC-A-2011-0696, the FPA2014-52823-C2-1-P and the SEV-2014-0398 Grants, by the European Commission under the FP7/EURATOM Grant, and by the Spanish Ministerio de Educación under the FPU program.

\section{References}

1] A. Algora, et al., Phys. Rev. Lett. 105 (2010) 202501.

[2] D. Jordan, et al., Phys. Rev. C 87 (2013) 044318.

[3] A. Algora, et al., Nuclear Data Sheets 120 (2014) 12.

[4] J. Hardy, et al., Phys. Lett. B 71 (2) (1977) 307.

[5] J. L. Tain, et al., Nucl. Instrum. and Methods A 803 (2015) 36.

[6] B. Rubio, Int. J. Modern Phys. E 15 (2006) 1979.

[7] T. Davinson, et al., Technical Report for the Design, Construction and Commissioning of the Advanced Implantation Detector Array (AIDA) [link] URL http://www.fair-center.eu/fileadmin/fair/ publications_exp/aida_TDR.pdf

[8] M. Fallot, et al., Phys. Rev. Lett. 109 (2012) 202504.

[9] K. L. Kratz, Nuclear Physics A 317 (1979) 335.

[10] J. L. Tain, et al., Phys. Rev. Lett. 115 (2015) 062502.

[11] V. S. Kolhinen, et al., Nucl. Instrum. and Methods B 317 (2013) 503. 
[12] T. Eronen, et al., Eur. Phys. J. A 48 (2012) 1.

[13] J. L. Tain, D. Cano-Ott, Nucl. Instrum. and Methods A 571 (2007) 728.

[14] D. Cano-Ott, et al., Nucl. Instrum. and Methods A 430 (1999) 333.

[15] A. Algora, et al., Phys. Rev. C 70 (2004) 064301.

[16] S. Agostinelli, et al., Nucl. Instrum. and Methods A 506 (2003) 250.

17] D. Cano-Ott, et al., Nucl. Instrum. and Methods A 430 (1999) 488.

[18] H. Ohm, et al., Z. Phys. A 296 (1980) 23.

[19] B. Fogelberg, et al., Phys. Rev. C 31 (1985) 2041.

[20] J. L. Tain, et al., Nucl. Instrum. and Methods A 774 (2015) 17

[21] E. Browne, J. K. Tuli, Nuclear Data Sheets 108 (2007) 2173. 